\title{
On the Validity of Nonlinear and Nonsmooth Inequalities
}

\author{
M. H. Noori Skandari \\ School of Mathematical Sciences, Shahrood University of Technology, Shahrood, Iran \\ E-mail: Math.Noori@yahoo.com
}

\begin{abstract}
In this article, a new approach is presented to survey the validity of the nonlinear and nonsmooth inequalities on a compact domain using optimization. Here, an optimization problem corresponding with the considered inequality is proposed and by solving of which, the validity of the inequality will be determined. The optimization problem, in s mooth and nonsmooth forms, is solved by a linearization approach. The efficiency of presented approach is illustrated in some examples.
\end{abstract}

Index Terms-Nonlinear and nonsmooth inequalities, Generalized Derivatives, Linearization approach, Smooth and Nons mooth Optimization.

\section{INTRODUCTION}

There are some techniques that are frequently employed in proving inequalities. A method to prove the inequalities is to start from one side of the inequality and apply a sequence of known inequalities to reach the other side, or we may start from both sides and try to reach a common point. In fact, few problems concerning inequalities can be proved by a direct application of one of the most important and well-known inequalities. So, it is important to apply known inequalities suitably in order that the desired result can be obtained. Also, sometimes we deal with unapparent inequalities.

Several generalizations and proofs are given related to the inequalities, such as Jensen type inequalities [1-4], Hermite-Hadamard inequality [5-10], etc. But, it should always be remembered that there is no standard way of proof and there is no general rule in choosing techniques to be used. Specially, when we deal with inequalities including complicated nonlinear or nonsmooth terms, the proof is very hard. Also, when the number of variables appeared in inequalities is great, we usually do not obtain the validity of inequalities and prove them.

In the recent decades, optimization techniques and methods are developed in many fields and problems, but we do not see a technique based on optimization to prove the inequalities or to survey their validity. Hence, in this paper, an approach is presented to investigate the validity of the following general inequality using optimization:

$$
F(X) \geq 0, \quad X \in \Omega
$$

where $F: \Omega \subset \mathbb{R}^{n} \rightarrow \mathbb{R}$ is a continuous function,
$X=\left(X_{1}, X_{2}, \ldots, X_{n}\right)$ and $\Omega$ is a compact subset of $\mathbb{R}^{n}$. Here, we do not know the inequality (1) is valid or invalid and there is not any approach to determine the validity of the general inequality (1).

\section{MAIN IDEA}

We consider the following problem corresponding to the inequality (1):

$$
\text { Minimize } F(X)
$$

We note that since function $F($.$) is continuous and \Omega$ is compact, the optimization problem (2) has an optimal solution on $\Omega$.

Theorem 2.1: Let $X^{*}$ be the optimal solution of the optimization problem (2). The inequality (1) is valid when $F\left(X^{*}\right) \geq 0$.

Proof: Since $X^{*}$ is the optimal solution of the optimization problem (2), for all $X \in \Omega$, we have $F(X) \geq F\left(X^{*}\right)$. So if $F\left(X^{*}\right) \geq 0$ then for all $X \in \Omega$ we have $F(X) \geq 0$, and this means inequality (1) is valid. Moreover, if $F\left(X^{*}\right)<0$ then inequality (1) is invalid.

By considering the Theorem 2.1, from solving the optimization problem (2), we can recognize the inequality (1) is valid or invalid.

There are several well-known methods and algorithms for solving optimization problem (2), such as line search method, gradient method, Quasi-Newton Method, linearization method, steepest descent method, BFGS Method, etc. In the following, a good linearization method is given to solve the problem (2) in two cases: the function $F($.$) is smooth (continuously differentiable) or$ nonsmooth (nondifferentiable). By this method, we can obtain an approximate global optimal solution for smooth or nonsmooth optimization problem (2).

\section{A. Linearization approach in order to solve the smooth optimization problem}


In this subsection, assume that function $F($.$) has the$ continuous second order derivative. Divide the set $\Omega_{\text {s }}$ to the similar grids $\Omega_{j}, j=1,2, \ldots, m$ such that $\Omega=\bigcup_{j=1}^{m} \Omega_{j}$ and for all $i \neq j, \operatorname{int}\left(\Omega_{i}\right) \bigcap \operatorname{int}\left(\Omega_{j}\right)=\Phi$ (where for every set $A$, the int $(A)$ shows the interior points of set $A \quad$ ). Select the arbitrary points $Z_{j} \in \operatorname{int}\left(\Omega_{j}\right), j=1,2, \ldots, m$, where $m$ is a sufficiently big number. By Taylor expansion, for all $X \in \Omega_{j}$, we have:

$$
\begin{aligned}
F(X) & =F\left(Z_{j}\right)+\sum_{i=1}^{n}\left(X_{i}-Z_{i j}\right) \frac{\partial F}{\partial X_{i}}\left(Z_{i j}\right) \\
& +O\left(\left\|X-Z_{j}\right\|^{2}\right), \quad j=1,2, \ldots, m
\end{aligned}
$$

where

$$
Z_{j}=\left(Z_{1 j}, Z_{2 j}, \ldots, Z_{n j}\right)
$$

$X=\left(X_{1}, X_{2}, \ldots, X_{n}\right)$. We can write for $j=1,2, \ldots, m$ :

$$
F(X)=\sum_{j=1}^{m}\left(\bar{F}(X)+O\left(\left\|X-Z_{j}\right\|^{2}\right)\right) I_{\Omega_{j}}(X)
$$

where

$$
\bar{F}(X)=F\left(Z_{j}\right)+\sum_{i=1}^{n}\left(X_{i}-Z_{i j}\right) \frac{\partial F}{\partial X_{i}}\left(Z_{i j}\right) .
$$

In (4), $I_{\Omega_{j}}(X)=1$ if, $X \in \Omega_{j}$ and in otherwise $I_{\Omega_{j}}(X)=0$. By relation (4), we have

$$
\begin{aligned}
\underset{X \in \Omega}{\operatorname{Minimum}} & F(X)= \\
& \sum_{j=1}^{m}\left(\bar{F}(X)+O\left(\left\|X-Z_{j}\right\|^{2}\right)\right) I_{\Omega_{j}}(X) .
\end{aligned}
$$

But, by attention to the selected sets $\Omega_{j}, j=1,2, \ldots, m$ and points $Z_{j}, j=1,2, \ldots, m$, there exists sufficiency big number $m_{0} \in \mathbb{N}$ such that for all $m \geq m_{0}, O\left[\left\|X-Z_{j}\right\|^{2}\right] \leq \frac{1}{m}, X \in \Omega_{j}$ and hence, for sufficiently great number $m$, we can ignore the term $O\left[\left\|X-Z_{j}\right\|^{2}\right], X \in \Omega_{j} \quad$ for $\quad$ all $j=1,2, \ldots, m$. Hence, the minimization problem (2) can be converted to the following minimization:

$$
\underset{X \in \Omega}{\operatorname{Minimize}} \sum_{j=1}^{m} \bar{F}(X) I_{\Omega_{j}}(X) \text {, }
$$

where $m$ is a sufficiently big number and function $\bar{F}($.$) is defined by (5). Thus, by solving nonlinear$ programming (NLP) problem (6), we can reach to an approximate optimal solution for (2). In below, is shown that by solving some linear programming (LP) problem, we can obtain the optimal solution of NLP problem (6).

Theorem 2.2: Let $X^{j,{ }^{*}}(j=1,2, \ldots, m)$ be the optimal solution of the following linear programming (LP) problem:

$$
\underset{X \in \Omega_{j}}{\operatorname{Minimize}} \bar{F}(X)=F\left(Z_{j}\right)+\sum_{i=1}^{n}\left(X_{i}-Z_{i j}\right) \frac{\partial F}{\partial X_{i}}\left(Z_{i j}\right)
$$

where $Z_{j}=\left(Z_{1 j}, Z_{2 j}, \ldots, Z_{n j}\right) \in \Omega_{j} \quad$ is given and $X=\left(X_{1}, X_{2}, \ldots, X_{n}\right)$ is the variable of the problem. Further, suppose that

$$
\bar{F}\left(X^{p, *}\right)=\underset{j=1,2, \ldots, m}{\operatorname{Minimum}} \bar{F}\left(X^{j,{ }^{*}}\right)
$$

Then $X^{p{ }^{*}}$ is a global optimal solution for the NLP problem (6).

Proof: Let $\bar{X} \in \Omega$ be an optimal solution for the NLP problem (6), $\bar{X} \neq X^{p,{ }^{*}}$ and $\bar{F}(\bar{X})<\bar{F}\left(X^{p,{ }^{*}}\right)$. So there is $k \in\{1,2, \ldots, m\}$ such that $\bar{X} \in \Omega_{k}$ and

$$
\begin{gathered}
\bar{F}\left(X^{k, *}\right)=\underset{X \in \Omega_{k}}{\operatorname{Minimum}} \bar{F}(X), \\
\bar{F}(\bar{X})=\underset{X \in \Omega}{\operatorname{Minimum}} \bar{F}(X) .
\end{gathered}
$$

But $\Omega_{k} \subset \Omega$, so $\bar{F}\left(X^{p,{ }^{*}}\right)=\operatorname{Minimum} \bar{F}\left(X^{j,{ }^{*}}\right)$. Hence,

$$
\bar{F}\left(X^{k, *}\right)=\bar{F}(\bar{X})
$$

On the other hand, $\bar{F}\left(X^{p, *}\right)=\underset{j=1,2, \ldots, m}{\operatorname{Minimum}} \bar{F}\left(X^{j, *}\right)$ and

$$
\bar{F}\left(X^{p, *}\right) \leq \bar{F}\left(X^{k, *}\right)
$$


By (8) and (9), we have $\bar{F}\left(X^{p, *}\right) \geq \bar{F}\left(X^{k, *}\right)$ which is a contradiction.

Remark 2.1: By Theorem 2.2, we solve the LP problem (7), for $j=1,2, \ldots m$, to obtain the optimal solution of the NLP problem (6). We note that, for sufficiently number $m$, the optimal solution of the NLP problem (6) is an approximate optimal solution for the NLP problem (2). Moreover, by Theorem 2.1, the optimal solution of the optimization problem (2) shows that smooth inequality (1) is valid or invalid.

B. Linearization approach in order to solve the nonsmooth optimization problem

We suppose that function $F($.$) is continuous but$ nonsmooth (or nondifferentiable) on W. Hence, the problem (2) is a nonsmooth optimization. In this paper, the practical generalized derivatives (GDs) and generalized first order Taylor expansion (FOTE) of nonsmooth functions proposed by Noori Skandari et.al. (see [11,12]) is used to solve the nonsmooth optimization problem (2). A similar approach is given [13] to solve the nonsmooth optimization problems. Also, some other types of GDs are presented in [14,15].

As the following, the GDs and generalized FOTE are introduced at first, and then they are utilized to solve the nonsmooth optimization problems (1).

Assume $C(\Omega)$ is the spaces of continuous and continuous differentiable functions on the set $\Omega$. Let $\varphi(),. j=0,1,2, \ldots$ are the continuously differentiable basic functions for the space $C(\Omega)$ and suppose $N_{\delta}(S)$ is the neighborhood of $S$ with the radius $\delta$. In addition, selecting the arbitrary points $S_{i} \in \operatorname{int}\left(\Omega_{\text {q }}\right)$ $i=1,2, \ldots, m$ where the sets $\Omega_{i}=(i=1,2, \ldots, m)$ are defined in subsection 2.1. Now, consider the following optimization problem:

$$
\begin{aligned}
& \underset{\mathrm{a}_{\mathrm{jk}} \in \mathbb{R}}{\operatorname{Minimize}} \\
& \sum_{i=1}^{m} \int_{N_{\delta}\left(S_{i}\right)}\left|\begin{array}{c}
F(X)-F\left(S_{i}\right)- \\
\sum_{k=1}^{n} \sum_{j=0}^{\infty}\left(X_{k}-S_{i k}\right) a_{j k} \varphi_{j}\left(S_{i}\right)
\end{array}\right| d X
\end{aligned}
$$

where $\delta>0$ is a given sufficiently small number, $X=\left(X_{1}, \ldots, X_{n}\right)$ and $S_{i}=\left(S_{i 1}, \ldots, \mathrm{S}_{i n}\right) \in \operatorname{int}\left(\Omega_{i}\right)$ for all $i=1,2, \ldots, m$. By assumption

$$
\eta_{k}(S)=\sum_{j=0}^{\infty} a_{j k} \varphi_{j}(S), \quad S \in \Omega, k=1,2, \ldots, n,
$$

$k=1,2, \ldots, n$, the problem (10) is equivalent to the following problem:

$$
\begin{aligned}
& \underset{\eta_{k}(.) \in C(\Omega)}{\operatorname{Minimize}} \\
& \sum_{i=1}^{m} \int_{N_{\delta}\left(S_{i}\right)}\left|\begin{array}{c}
F(X)-F\left(S_{i}\right)- \\
\sum_{k=1}^{n}\left(X_{k}-S_{i k}\right) \eta_{k}\left(S_{i}\right)
\end{array}\right| d X
\end{aligned}
$$

Now, let $\left(\eta_{1}^{*}(),. \ldots, \eta_{n}^{*}().\right)$ be the optimal solution of the optimization problem (11). The GD of the function $F($.$) with respect to X_{k}$ is denoted by $\partial_{X_{k}} F($.) and defined as $\partial_{X_{k}} F\left(S_{i}\right)=\eta_{k}^{*}\left(S_{i}\right)$ for $k=1,2, \ldots, n$ and $i=1,2, \ldots, m$ (see $[6,7])$. Here, by assumption

$$
\eta_{k}(S)=\sum_{j=0}^{M} a_{j k} \varphi_{j}(S), S \in \Omega, k=1,2, \ldots, n
$$

(where $M$ is a sufficiently big number), the infinite dimensional nonsmooth optimization problem (11) can be approximated to the following finite dimensional smooth problem (see [12]):

$$
\underset{v(. .,), a_{j k}}{\operatorname{Minimize}} \sum_{i=1}^{m} \int_{N_{\delta}\left(S_{i}\right)} v\left(X, S_{i}\right) d X
$$

subject to

$$
\begin{aligned}
& -v\left(X, S_{i}\right) \leq F(X)-F\left(S_{i}\right) \\
& -\sum_{k=1}^{n} \sum_{j=0}^{M}\left(X_{k}-S_{i k}\right) a_{j k} \varphi_{j}\left(S_{i}\right), \\
& -v\left(X, S_{i}\right) \leq-F(X)+F\left(S_{i}\right) \\
& +\sum_{k=1}^{n} \sum_{j=0}^{M}\left(X_{k}-S_{i k}\right) a_{j k} \varphi_{j}\left(S_{i}\right), \\
& i=1,2, \ldots, m, X \in \Omega .
\end{aligned}
$$

Further, in $[11,12]$ is showed that this smooth problem can be approximated to a LP problem. Hence, the GD of nonsmooth function $F($.$) is as$ $\partial_{X_{k}} F()=.\sum_{j} a_{j k}^{*} \varphi_{j}(),. k=1,2, \ldots, n \quad$ where $a_{j k}^{*}$ (for all $k$ and $j$ ) is the optimal solution of the linear optimization problem (12). In [12] is showed that the best linear approximation of the continuous nonsmooth function $F($.$) , in passing of point S_{i} \in \operatorname{int}(\Omega)$, is as follows: 


$$
\begin{gathered}
\bar{F}(X)=F\left(S_{i}\right)+\sum_{k=1}^{n}\left(X-S_{i k}\right) \partial_{X_{k}} F\left(S_{i}\right), \\
X \in N_{\delta}\left(S_{i}\right)
\end{gathered}
$$

where $\partial_{X_{k}} F\left(S_{i}\right)=\sum_{j=0}^{M} a_{j}^{k} \varphi_{j}\left(S_{j}\right)$ for $k=1,2, \ldots, n$ and, $a_{j}^{k}$ for $j=0,1, \ldots, M$ and $k=1,2, \ldots, n$ are the optimal solutions of the problem (12). Further, in [12] is showed that the total error $\bar{E}_{\delta, \partial F(s)}()=.\|F()-.\bar{F}().\| N_{\delta}(s) \quad$ of $\quad$ the approximation $F(.) \simeq \bar{F}\left(\right.$.) on $N_{\delta}(S)$, tends to zero when $\delta \rightarrow 0$. Also, the generalized FOTE of the continuous nonsmooth function $F: \Omega \subset \mathbb{R}^{n} \rightarrow \mathbb{R}$ is given as follows:

$$
\begin{aligned}
F(X)= & F\left(S_{i}\right)+\sum_{k=1}^{n}\left(X_{k}-S_{i k}\right) \partial_{x_{k}} F\left(S_{i}\right) \\
& +E_{\delta, \partial F\left(S_{i}\right)}(X), \quad X \in N_{\delta}\left(S_{i}\right),
\end{aligned}
$$

where $E_{\delta, \partial f\left(S_{i}\right)}(X)$ is the pointwise error of the approximation $F(X) \approx \bar{F}(X), \quad X \in N_{\delta}\left(S_{i}\right)$ (the function $\bar{F}($.$) is defined by (13)).$

By generalized FOTE, the minimization problem (2) lead to the following minimization:

$$
\underset{X \in \Omega}{\operatorname{Minimize}} \sum_{i=1}^{m} \bar{F}(X) I_{\Omega_{i}}(X)
$$

where $m$ is a given sufficiently big number, linear function $\bar{F}\left(\right.$.) is defined by (13), and $I_{\Omega i}($.$) is$ characteristic function of set $\Omega_{i}$.

Theorem 2.3: Consider the NLP problem (15). Let $X^{i, *}(i=1,2, \ldots, m)$ be the optimal solution of the following linear programming (LP) problem:

$$
\begin{aligned}
& \underset{X \in \Omega_{i}}{\operatorname{Minimize}} \\
& \bar{F}(X)=F\left(S_{i}\right)+\sum_{j=1}^{n}\left(X_{j}-S_{i j}\right) \partial_{X_{j}} F\left(S_{i j}\right),
\end{aligned}
$$

where $\quad S_{i}=\left(S_{i 1}, \ldots, S_{i n}\right) \quad$ is $\quad$ given, $X=\left(X_{1}, X_{2}, \ldots, X_{n}\right)$ is the variable of the problem, and $\partial_{X_{j}} F($.$) is the GD of F($.$) with respect to$
$X=\left(X_{1}, X_{2}, \ldots, X_{n}\right)$. Further, suppose that

$$
\bar{F}\left(X^{p, *}\right)=\underset{i=1,2, \ldots, m}{\operatorname{Minimum}} \bar{F}\left(X^{i, *}\right) .
$$

Then $X^{p{ }^{*}}$ is the global optimal solution of the NLP problem (15).

Proof: The proof is similar to the proof of Theorem 2.2.

Remark 2.2: By above theorem, we solve the LP problem (16), for $i=1,2, \ldots m$, to obtain the optimal solution of the NLP problem (15), and then obtain an approximate optimal solution for the nonsmooth optimization problem (2) to determine the validity of nonsmooth inequality (1).

\section{SIMULATION RESULTS}

In this section, the results and efficiency of presented approach be simulated in some examples.

Example 3.1: We survey the validity of the following smooth inequality:

$$
\begin{array}{r}
e^{x+1}+\operatorname{Ln}\left(x^{2}+1\right) \geq x^{2}+2 \sin (\pi x) \\
+2 \cos (\pi x), \quad x \in[0,1] .
\end{array}
$$

We define

$$
\begin{aligned}
F(x) & =e^{x+1}+\operatorname{Ln}\left(x^{2}+1\right) \\
& -x^{2}-2 \sin (\pi x)-2 \cos (\pi x), x \in[0,1],
\end{aligned}
$$

and solve the NLP problem

$$
\begin{array}{lc}
\text { Minimize } & F(x) \\
\text { subject to } & 0 \leq x \leq 1 .
\end{array}
$$

For applying the linearization approach stated in Section 1.2, we assume that $m=1000$ and $\Omega_{j}=\left[\frac{j-1}{m}, \frac{j}{m}\right], j=1,2, \ldots, m$ and select points $z_{j}=\frac{2 j-1}{2 n}, j=1,2, \ldots, m$ on $\operatorname{int}\left(\Omega_{j}\right)$ Now, the corresponding LP problem (7) for $j=1,2, \ldots, m$ is as follows:

$$
\begin{array}{ll}
\text { Minimize } & \bar{F}(x)=F\left(z_{j}\right)+\left(x-z_{j}\right) F^{\prime}\left(z_{j}\right) \\
\text { subject to } & \frac{j-1}{m} \leq x \leq \frac{j}{m},
\end{array}
$$


where

$$
\begin{aligned}
& F^{\prime}\left(z_{j}\right)=e^{z_{j}+1}+\frac{2 z_{j}}{\left(z_{j}^{2}+1\right)}-2 z_{j} \\
& -2 \pi \cos \left(\pi z_{i}\right)+2 \pi \sin \left(\pi z_{i}\right), \quad j=1,2, \ldots, m .
\end{aligned}
$$

By solving the LP problem (19), we obtain the optimal solution $x^{j, *}, j=1,2, \ldots, m$. Moreover, we have

$$
\underset{j=1,2, \ldots, m}{\operatorname{Minimum}} \bar{F}\left(x^{j, *}\right)=\bar{F}\left(x^{p,{ }^{*}}\right)=0.4651542 .
$$

where $x^{p,{ }^{*}}=0.1360000$ is an approximate global optimal solution for the smooth NLP problem (20). Thus, by attending to Remark 1.2, since $F(x) \geq 0.4651505>0$ the inequality (18) is valid. Here, the graph of functions $F($.$) and F^{\prime}($.$) are$ showed in Figure 1.

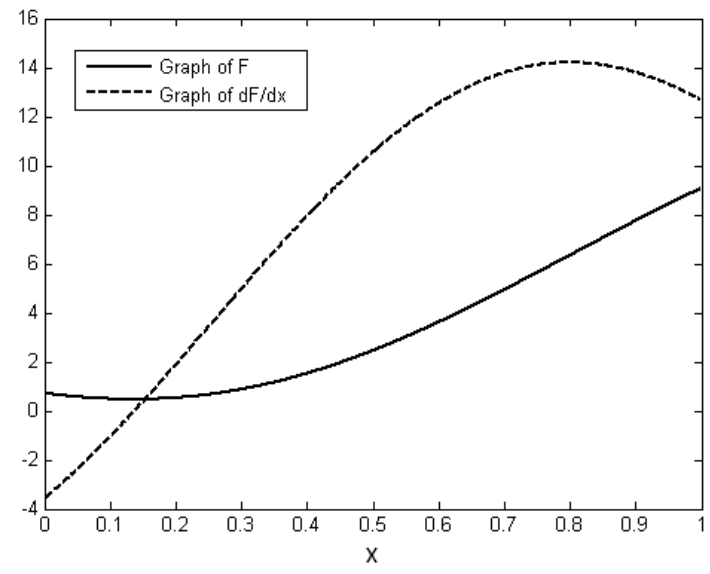

Fig.1. Graph of function $F($.$) and its GD in Example 3.1.$

Example 3.2: We show that the following nonsmooth inequality is invalid:

$$
2|x-0.5|+5 x \geq e^{|x-0.25|}, \quad x \in[0,1] .
$$

We define

$$
F(x)=2|x-0.5|+5 x-e^{|x-0.25|}, \quad x \in[0,1],
$$

and assume that $m=1000$ and $\Omega_{i}=\left[\frac{i-1}{m}, \frac{i}{m}\right]$, $i=1,2, \ldots, m$. Also, we select points $s_{i}=\frac{2 i-1}{m}$, $i=1,2, \ldots, m-1$ on $\operatorname{int}\left(\Omega_{f}\right)$. We first calculate the GD of nonsmooth function $F($.$) defined by (21). For$ this goal, we discrete the corresponding continuous optimization problem (12) the same as works [11,12]. After this discretization, we reach to a LP problem and by solving of which we obtain GD of $F($.$) as$ $\partial_{x} F\left(s_{i}\right), i=1,2, \ldots, m$, where it is illustrated in Figure

2. Now, we solve the nonsmooth NLP problem

Minimize $F(x)=2|x-0.5|+5 x-e^{|x-0.25|}$

$$
\text { Subject to } 0 \leq x \leq 1
$$

Here, the corresponding LP problem (16) for $i=1,2, \ldots, m$ is as follows:

$$
\begin{array}{cl}
\text { Minimize } & \bar{F}(x)=F\left(s_{j}\right)+\left(x-s_{j}\right) \partial_{x} F\left(s_{i}\right) \\
\text { subject to } \frac{i-1}{m} \leq x \leq \frac{i}{m}
\end{array}
$$

By solving the LP problem (23), we obtain the optimal solution $x^{j, *}, j=1,2, \ldots, m$. Moreover, we have

$$
\begin{aligned}
\underset{j=1,2, \ldots, m}{\operatorname{Minimum}} \bar{F}\left(x^{j, *}\right) & =\bar{F}\left(x^{p,{ }^{*}}\right) \\
& =-0.2840252<0
\end{aligned}
$$

where $x^{p{ }^{*}}=0.5 \times 10^{-16}$ is an approximate global optimal solution for the nonsmooth NLP problem (22). Thus, by attending to the Remark 2.2, the inequality (20) is invalid.

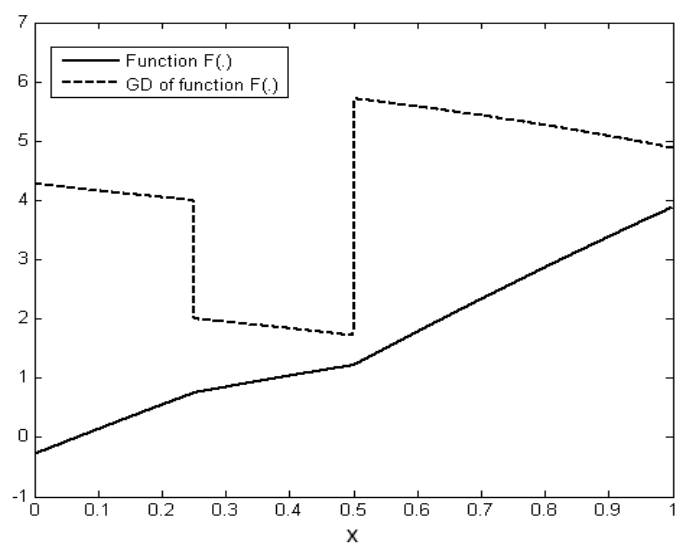

Fig.2. Graph of function $F($.$) and its GD in Example 3.2.$

Example 3.3: We survey the validity of the following nonsmooth inequality:

I.J. Intelligent Systems and Applications, 2017, 1, 60-66 


$$
\begin{aligned}
& 3 x_{1} x_{2}+2\left|x_{1}-x_{2}\right|+0.5 \geq \\
& 1.5 x_{2}+x_{1} \mid \sin \left(\pi\left(x_{2}-0.75\right) \mid, x_{1}, x_{2} \in[0,1]^{2} .\right.
\end{aligned}
$$

Assume that

$$
\begin{gathered}
F\left(x_{1}, x_{2}\right)=3 x_{1} x_{2}+2\left|x_{1}-x_{2}\right| \\
-1.5 x_{2}-x_{1} \mid \sin \left(\pi\left(x_{2}-0.75\right) \mid+0.5,\right. \\
x_{1}, x_{2} \in[0,1]^{2}
\end{gathered}
$$

and solve the following nonsmooth NLP problem:

$$
\begin{array}{ll}
\text { Minimize } & F\left(x_{1}, x_{2}\right) \\
\text { subject to } & 0 \leq x_{1} \leq 1,0 \leq x_{2} \leq 1 .
\end{array}
$$

For this purpose, we assume that $m=200$ and $\Omega_{i j}=\left[\frac{i-1}{m}, \frac{i}{m}\right] \times\left[\frac{j-1}{m}, \frac{j}{m}\right]$, for $i=1,2, \ldots, m$ and $j=1,2, \ldots, m \quad$. Also, select points $\left(s_{i}, s_{j}\right)=\left(\frac{2 i-1}{m}, \frac{i}{m}\right] \times\left[\frac{2 j-1}{m}, \frac{j}{m}\right) \in \operatorname{int}\left(\Omega_{i j}\right)$. We first calculate the GD of nonsmooth function $F($.) defined by (25) where it is illustrated in Figure 3. By discretization the corresponding continuous optimization problem (16), we reach to the some LP problems and obtain the GD of $F($.$) with respect to x_{1}$ and $x_{2}$ as $\partial_{x 1} F\left(s_{i}, s_{j}\right)$ and $\partial_{x 2} F\left(s_{i}, s_{j}\right)$, for $i=1,2, \ldots, m$ and $j=1,2, \ldots, m$, where they are illustrated in Figures 4 and 5, respectively. Now, the corresponding LP problem (16), for solving the nonsmooth NLP problem (26), is as follows (for $i=1,2, \ldots, m$ and $j=1,2, \ldots, m)$ :

$$
\begin{aligned}
\operatorname{Minimize} & \bar{F}\left(x_{1}, x_{2}\right)=F\left(s_{i}, s_{j}\right) \\
& +\left(x_{1}-s_{i}\right) \partial_{x_{1}} F\left(s_{i}, s_{j}\right) \\
& +\left(x_{2}-s_{j}\right) \partial_{x_{2}} F\left(s_{i}, s_{j}\right)
\end{aligned}
$$

subject to $\frac{i-1}{m} \leq x_{1} \leq \frac{i}{m}, \frac{j-1}{m} \leq x_{2} \leq \frac{j}{m}$,

By solving the LP problem (27), we obtain the optimal solution $\left(x_{1}^{i^{*}}, x_{2}{ }^{j^{*}}\right)$ for $i=1,2, \ldots, m$ and $j=1,2, \ldots, m$. Moreover, we have

$$
\begin{aligned}
\underset{i, j=1,2, \ldots, m}{\operatorname{Minimum}} \bar{F}\left(x_{1}^{i^{*}}, x_{2} j^{*}\right) & =\bar{F}\left(x_{1}^{p^{*}}, x_{2}^{q^{*}}\right) \\
& =0.0004391>0
\end{aligned}
$$

where $\left(x_{1}^{p^{*}}, x_{2}^{q^{*}}\right)=(0.3524999,3524999)$ is an approximate global optimal solution for the nonsmooth NLP problem (26). Thus, by attending to the Remark 2.2, the inequality (24) is valid.

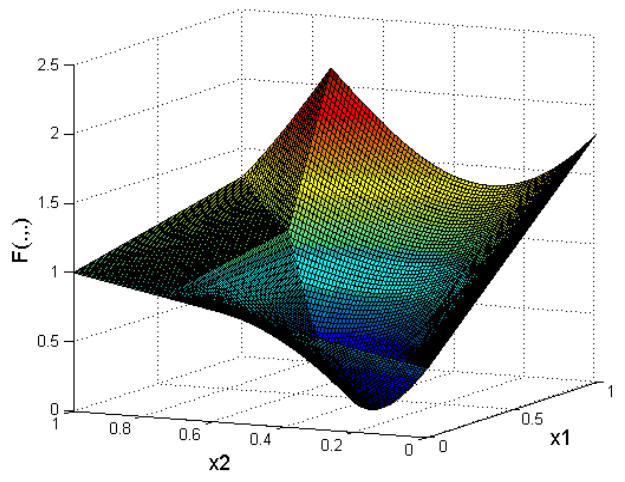

Fig.3. The graph of function $F(.,$.$) for Example 3.3.$

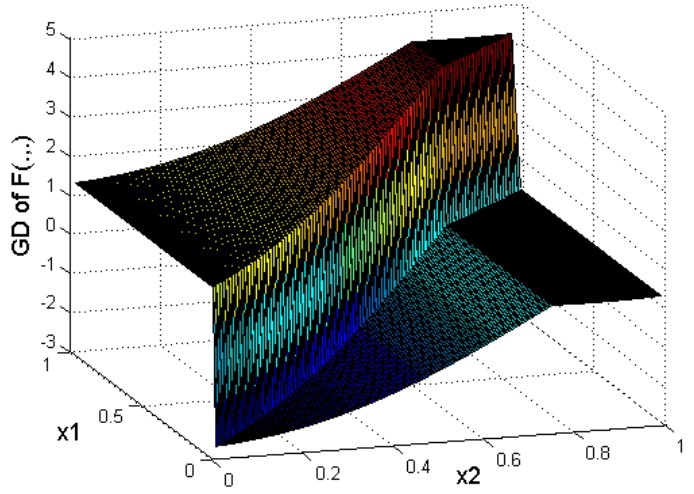

Fig.4. The graph of $\partial_{x 1} F(.,$.$) for Example 3.3.$

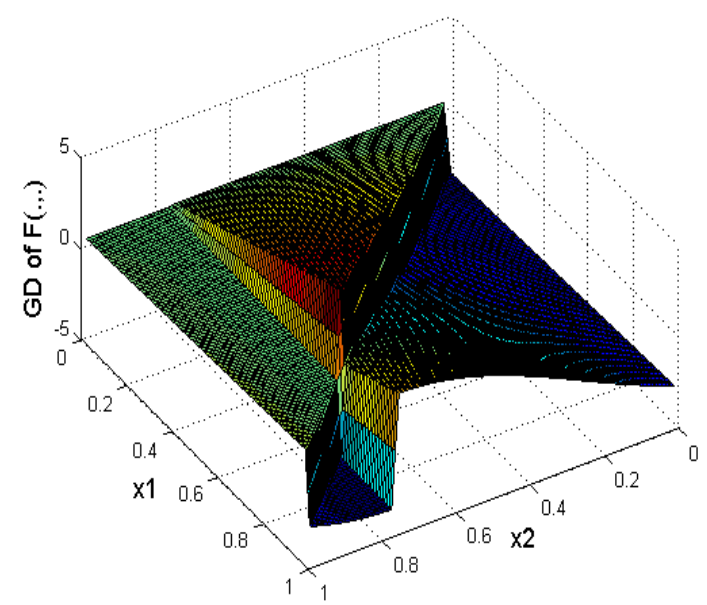

Fig.5. The graph of $\partial_{x 2} F(.,$.$) for Example 3.3$ 


\section{CONCLUSION}

The nonlinear and nonsmooth inequalities can be proved or rejected by applying a s mooth or nonsmooth optimization problems. In fact, we can solve an optimization problem and receive to the validity of inequality. This optimization problem can be solved by optimization techniques such as linearization approach, approximately and globally.

\section{REFERENCES}

[1] A. Matkovic and J. Pecaric, A Variant of Jensen's inequality for convex functions of several variables, Journal of Mathematical Inequalities, 1(2007), 1, 45-51.

[2] J. I. Fujii, J. Pecaric and Y. Seo, The Jensen inequaly in an external formula, Journal of Mathematical Inequalities, 6(2012), 3, 473-480.

[3] L. Horvath, A new refinement of discrete Jensen's inequality depending on parameters, Journal of Inequalities and Applications, 551(2013).

[4] A. R. Khan, J. Pecaric Ecaric and M. R. Lipanovic, NExponential Convexity for Jensen-type Inequalities, Journal of Mathematical Inequalities, 7(2013), 3, 313335.

[5] C. G. Cao, Z. P.Yang, X. Zhang, Two inequalities involving Hadamard products of positive semi-definite Hermitian matrices, Journal of Applied Mathematics and Computing , 10(2002)1-2, 101-109.

[6] T. Trif, Charactrizations of convex functions of a vector variable via Hermit-Hadmard's inequality, Journal of Mathematical Inequalities, 2(2008), 1,37-44.

[7] Y. Wang, M. M Zheng, F. Qi , Integral inequalities of Hermite-Hadamard type for functions whose derivatives are $\alpha$-preinvex, Journal of Inequalities and Applications 97(2014).

[8] S. H. Wang, F. Qi , Hermite-Hadamard type inequalities for $n$-times differentiable and preinvex functions, Journal of Inequalities and Applications , 49(2014).

[9] C. J Zhao, W. S. Cheung, X.Y. Li , On the HermiteHadamard type inequalities, Journal of Inequalities and Applications, 228 (2013).

[10] X. Gao, A note on the Hermite-Hadamard inequality, Journal of Mathematical In equalities, 4(2010), 4, 587-591.

[11] M. H. Noori Skandari, A.V. Kamy ad and H. R. Erfanian, A New Practical Generalized Derivative for Nonsmooth Functions, The Electronic Journal of Mathematics and Technology, 7(2013),1.

[12] M. H. Noori Skandari, A. V. Kamyad and S. Effati, Generalized Euler-Lagrange Equation for Nonsmooth Calculus of Variations, Nonlinear Dyn., 75(2014), 85-100.

[13] H. R. Erfanian, M. H. Noori Skandari, and A. V. Kamyad, A Novel Approach for Solving Nonsmooth Optimization Problems with Application to Nonsmooth Equations, Hindawi Publishing Corporation, Journal of Mathematics, Vol. 2013, Article ID 750834.

[14] H. R. Erfanian, M. H. Noori skandari and A. V. Kamayad, A New Approach for the Generalized First Derivative and Extension It to the Generalized Second Derivative of Nonsmooth Functions, I.J. Intelligent Systems and Applications, 04 (2013), 100-107.

[15] M. H. Noori Skandari, H. R. Erfanian, A. V. Kamy ad and M. H. Farahi, Solving a Class of Non-Smooth Optimal Control Problems, I.J. Intelligent Systems and Applications,07( 2013) 07, 16-22.

\section{Authors' Profiles}

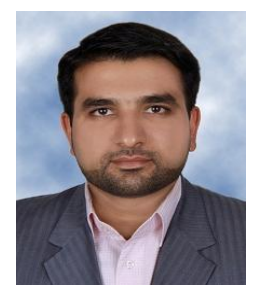

Mohammad Hadi Noori Skandari Received his B.Sc., M.Sc. and Ph.D. degrees in Applied Mathematics from Ferdowsi University of Mashhad, M ashhad, Iran, in 2005, 2007 and 2012 respectively. $\mathrm{He}$ is an Assistant Professor in Applied Mathematics in Faculty of Mathematics at Shahrood University of Technology, Shahrood, Iran. His research interests include Optimal Control, Optimization, Numerical Methods, Nonsmooth systems, Control Systems, Fuzzy Sy stems and Modeling.

How to cite this paper: M. H. Noori Skandari,"On the Validity of Nonlinear and Nonsmooth Inequalities", International Journal of Intelligent Systems and Applications(IJISA), Vol.9, No.1, pp.60-66, 2017. DOI: 10.5815/ijisa.2017.01.06 